and the strategy for that is early intervention and early identification of individuals at risk. When you have an integrated health system, you have a much better opportunity to do that, because all members of the care team have a way of communicating, for example, using the electronic health record.'

The National Health Service in the UK has had all these systems in place for some time - where do we go wrong?

Magellan Health - Suicide Prevention Programme. Presentation at the annual meeting of the American Psychiatric Association, 15 May 2016.

\section{Magic mushrooms for depression?}

$\mathrm{L}$ SD and other hallucinogenic drugs were inUvestigated as potential therapeutic agents in the $1960 \mathrm{~s}$, but interest declined over the following decades, only to resurge in the past few years. Psilocybin, a component of some species of magic mushrooms, is a $5 \mathrm{HT}_{2 \mathrm{~A}}$ serotonin receptor agonist and a hallucinogenic agent. It was given to patients with treatment-resistant unipolar depression in an open-label study of 6 men and 6 women, and an improvement in symptoms was noted at 1 week and at up to 3 months after treatment.

This is a very small study with several limitations and no conclusions can be drawn about the potential usefulness of such agents in the treatment of depression or indeed about its safety. Perhaps the emphasis should be on using such hallucinogenic agents to try to develop a better understanding of the workings of the brain and the mechanisms involved in the genesis of symptoms, before going on to clinical trials with patients?

Carhart-Harris, R. L., Bolstridge, M., Rucke, ], et al (2016)

Psilocybin with psychological support for treatment-resistant depression: an open-label feasibility study. Lancet Psychiatry. doi: 10.1016/S2215-0366(16)30065-7.

\section{There is light at the end of the tunnel!}

Dhototherapy has established itself as an effective P treatment for seasonal affective disorder and it is popular with patients. Although it has been considered also in the treatment of non-seasonal depression, there hasn't been sufficient interest in this. In a recently reported study, patients with major depressive disorder of moderate severity were randomised to treatment over 8 weeks with: (1) light monotherapy (30 minutes) plus placebo pill; (2) antidepressant monotherapy (20 mg fluoxetine) plus an inactive negative ion generator (30 minutes); (3) light plus fluoxetine; and (4) placebo light and placebo pill. Light therapy alone or in combination was effective, although the combination treatment had the most consistent results.

Lam, R. W., Levitt, A. J., Levitan, R. D., et al (2016) Efficacy of bright light treatment, fluoxetine, and the combination in patients with non-seasonal major depressive disorder. A randomized clinical trial. JAMA Psychiatry, 73, 56-63. doi: 10.1001/ jamapsychiatry.2015.2235.

\section{Agitation and psychosis in dementia - to treat or not to treat?}

$\mathrm{T}$ here has been increasing concern for some years about the use of second-generation antipsychotic drugs (SGAs) in people with dementia, with evidence emerging that these are associated with an increased risk of strokes in this population. In October 2009, a report for the Minister of State for Care Services by Professor Sube Bannerjee, commissioned and funded by the Department of Health, recommended a drastic reduction in their use, based on existing research evidence.

A recently published study disputes previous findings. The authors conducted a meta-analysis of population-based studies to assess the risk of stroke in dementia patients prescribed SGAs rather than the FGAs (first-generation antipsychotics). They found no differences in the risk of stroke between the SGAs and FGAs. This doesn't mean there is no risk!

The reader may be interested in the new practice guidelines on the use of antipsychotics in dementia issued by the American Psychiatric Association (http://psychiatryonline.org/doi/book/10.1176/appi. books.9780890426807).

Rao, A., Suliman, A., Story, G., et al (2016) Meta-analysis of population-based studies comparing risk of cerebrovascular accident associated with first- and second-generation antipsychotic prescribing in dementia. International Journal of Methods in Psychiatric Research. doi: 10.1002/mpr.1509. [Epub ahead of print]

\section{Watch too much television at your peril!}

$\Delta$ lot has been said about sedentary life and Aobesity and heart disease, but what about brain health? The authors of a prospective study of over 3000 adults (aged 18-30 years) who had enrolled in the Coronary Artery Risk Development in Young Adults (CARDIA) Study (1985-2011) investigated a possible association between patterns of TV viewing and physical activity and mid-life cognition. They visited participants and carried out at least three assessments over 25 years. A high level of television viewing was defined as over 3 hours per day for over two-thirds of the visits. At baseline and at year 25, they assessed cognitive function using the Digit Symbol Substitution Test, Stroop Test and Rey Auditory Verbal Learning Test. The high television viewers with low physical activity were two times more likely to have poor cognitive performance.

You have been warned!

Hoang, T. D., Reis, J., Zhu, N., et al (2016) Effect of early adult patterns of physical activity and television viewing on midlife cognitive function. JAMA Psychiatry, 73, 73-79. doi:10.1001/ jamapsychiatry.2015.2468. 